\title{
The verbal width of acylindrically hyperbolic groups is infinite
}

\author{
Mladen Bestvina, Ken Bromberg and Koji Fujiwara*
}

March 9, 2021

\begin{abstract}
We show that the verbal width is infinite for acylindrically hyperbolic groups, which include hyperbolic groups, mapping class groups and $\operatorname{Out}\left(F_{n}\right)$.
\end{abstract}

\section{Verbal subgroups}

The Brooks construction is a method for constructing essential quasi-morphisms on free groups. These Brooks quasi-morphisms can be extended to general acylindrically hyperbolic groups and in this note we use these quasimorphisms to study verbal subgroups. A verbal subgroup generalizes the notion of a commutator subgroup. We begin with a precise definition. Suppose that $G$ is a group and $\mathbb{F}_{k}$ is the free group generated by $x_{1}, \cdots, x_{k}$. For any choice of $w \in \mathbb{F}_{k}$ substitution defines a map, also denoted $w$ :

$$
w: G^{k} \rightarrow G .
$$

In what follows we always assume that $w$ is a non-trivial.

The image of this function is the verbal subset, and is denoted by $w[G]$. The verbal subgroup $w(G)<G$ is the subgroup generated by $w[G]$. For example, if $k=2$ and $w=x_{1} x_{2} x_{1}^{-1} x_{2}^{-1}$ then $w[G]$ is the set of commutators in $G$ and $w(G)=[G, G]$ is the commutator subgroup of $G$.

${ }^{*}$ This material is based upon work supported by the National Science Foundation under Grant No. DMS-1440140 while the authors were in residence at the Mathematical Sciences Research Institute in Berkeley, California, during the Fall 2016 semester. The first two authors gratefully acknowledge the support by the National Science Foundation under grant numbers DMS-1607236 and DMS-1509171 respectively. The third author is supported in part by Grant-in-Aid for Scientific Research (No. 15H05739) 
Let $e_{i}$ be the sum of the exponents of $x_{i}$ in $w$. For example, if $w=$ $x_{2} x_{1} x_{2}^{-2}$, then $e_{1}=1, e_{2}=1-2=-1, e_{3}=\cdots=e_{k}=0$. Let $d(w) \geq 1$ be the g.c.d. of $e_{i}$ 's. If they are all 0 , define $d(w)=0$. Note that the condition that $d(w)=0$ implies that $w(G) \subset[G, G]$ for when $d(w)=0, w(G)$ will be in the kernel of any homorphism from $G$ to an abelian group.

For each $g \in w(G)$, define its verbal length by

$$
v l_{w}(g)=\min \left\{n \mid g=g_{1} \cdots g_{n}, g_{i} \in w[G]^{ \pm 1}\right\} .
$$

The width of $w(G)$ is the supremum of $v l_{w}(g)$ over all $g \in w(G)$. Note that if $d(w)>1$, then $g^{d} \in w[G]$. (Replace each $x_{i}$ in $w$ with $g^{a_{i} e_{i}}$ where $\sum a_{i} e_{i}=d$.) In particular if $d=1$ then $w[G]=w(G)=G$ and the width is 1. The reader can consult the book [10] for more information on the subject. If $w$ is the commutator $\left[x_{1}, x_{2}\right]$, the verbal length is called the commutator length.

A group $G$ is acylindrically hyperbolic if it has a non-elementary acylindrical action on a $\delta$-hyperbolic space [8]. Recall that an isometric action of $G$ on a metric space $X$ is acylindrical if for all $D>0$ there exist $L, N>0$ such that if $d(x, y)>L$ then the set

$$
\{g \in G \mid d(x, g x)<D \text { and } d(y, g y)<D\}
$$

has $<N$ elements. The first nontrivial example is due to Bowditch [3] who showed that the action of the mapping class group on the curve complex is acylindrical. There are now many examples with the key point being that many seemingly weaker geometric criteria imply that the group is acylindrically hyperbolic. See [8] and [2].

Here is our main result.

Theorem 1.1. Suppose that $G$ is acylindrically hyperbolic and that $d(w) \neq$ 1. Then the width of $w(G)$ is infinite.

This result generalizes the work of Rhemtulla [9] and Myasnikov-Nikolaev [7] who proved the theorem for free groups and hyperbolic groups, respectively.

Similarly to the stable commutator length, one can define the stable verbal length of $g \in w(G), \operatorname{svl}_{w}(g)$, as follows:

$$
s v l_{w}(g)=\liminf _{n \rightarrow \infty} \frac{v l_{w}\left(g^{n}\right)}{n} .
$$

If $\operatorname{svl}_{w}(g)>0$ for some $g$ then $w(G)$ have infinite verbal width. However, if $d(w) \geq 1$ this method cannot be used due to the following lemma: 
Lemma 1.2 (Calegari-Zhuang [4]). If $d=d(w) \geq 1$ then $v l_{w}\left(g^{n}\right)$ is bounded and $\operatorname{svl}_{w}(g)=0$ for all $g \in w(G)$.

We may supress $w$ and write $v l, s v l$ instead of $v l_{w}, s v l_{w}$.

Proof. As observed above, $g^{d}$ is in $w[G]$ for all $g \in G$ so $v l\left(g^{n d}\right)=1$. Since $v l(g h) \leq v l(g)+v l(h)$ this implies that $v l\left(g^{n}\right)$ is bounded and $\operatorname{svl}(g)=0$.

On the other hand, when $d(w)=0$ we have the following which implies Theorem 1.1 in this case.

Theorem 1.3. If $G$ is acylindrically hyperbolic and $d(w)=0$, then $\operatorname{svl}_{w}(g)>$ 0 for some element $g \in w(G)$.

If $G$ is a free group then this is Corollary 2.16 of [4].

\subsection{An outline of proof of Theorem 1.1 for free groups}

To illustrate the main idea, we sketch the proof of Theorem 1.1 in the case that $G=F$ is the free group with basis $\{a, b\}$ and $w=x_{1} x_{2} x_{1} x_{2}^{-1}$, so that $d=d(w)=2$. Consider $g_{i}=a b^{2 i} a b^{2 i+1} \in G$ for $i=1,2, \cdots$. This sequence has the property that distinct occurrences of any $g_{i}$ in any reduced word have trivial overlap. Denote by $H_{i}: G \rightarrow \mathbb{Z}$ the Brooks counting quasi-morphism with respect to $g_{i}$. For any $y, z \in G$ we have $\left|H_{i}(y z)-H_{i}(y)-H_{i}(z)\right| \leq 3$ by the usual tripod argument, since at most 3 copies of $g_{i}$ along a tripod can have the tripod point in the interior. The key observation now is that in fact $H_{i}(y z)-H_{i}(y)-H_{i}(z)=0$ for all but at most 3 values of $i$ by the non-overlapping property of the $g_{i}$ 's (the exceptional values of $i$ depend on $y$ and $z$ ).

Now suppose that $g \in G$ has $v l_{w}(g)=1$, so $g=x_{1} x_{2} x_{1} x_{2}^{-1}$ for some $x_{1}, x_{2} \in G$. Then

$$
H_{i}(g)=H_{i}\left(x_{1}\right)+H_{i}\left(x_{2}\right)+H_{i}\left(x_{1}\right)+H_{1}\left(x_{2}^{-1}\right)=2 H_{i}\left(x_{1}\right)
$$

is even for all but $3 \times 3=9$ values of $i$. Thus to detect $g$ with $v l_{w}(g)>1$ it suffices to ensure that $H_{i}(g)$ is odd for 10 values of $i$. Similarly, to detect that $v l_{w}(g)$ is large it suffices to ensure that $H_{i}(g)$ is odd for sufficiently many $i$. An element such as

$$
(a b)^{2}\left(a b^{2}\right)^{2}\left(a b^{3}\right)^{2}\left(a b^{4}\right)^{2} \cdots\left(a b^{N-1}\right)^{2}\left(a b^{N}\right)^{2}
$$

will do.

For a general acylindrically hyperbolic group $G$ we perform the above construction on a suitable Schottky subgroup $F \subset G$ and then use the 
method of Hull and Osin [6] to extend the quasi-morphism from $F$ to $G$. We will review their construction in Section 4 and show that the key observation above continues to hold for the extended quasi-morphisms.

\section{Extending Brooks quasi-morphisms to acylin- drically hyperbolic groups}

We first recall the definition of a quasi-morphism. Let $G$ be a group. Then

$$
H: G \rightarrow \mathbb{R}
$$

is a quasi-morphism if

$$
\sup _{\alpha, \beta \in G}|H(\alpha \beta)-H(\alpha)-H(\beta)|=\Delta(H)<\infty .
$$

The constant $\Delta=\Delta(H)$ is the defect of $H$. Note that if $\Delta=0$ then $H$ is a homomorphism. A quasi-morphism is anti-symmetric if $H(-\alpha)=-H(\alpha)$.

One way to construct a quasi-morphism that is not a homomorphism is to start with a homomorphism and then add on a bounded function. Of course, this is not an interesting example. The Brooks construction is a way of building an anti-symmetric quasi-morphisms that are not a bounded distance from a homomorphism.

Let $F=\langle a, b\rangle$ be the free group on two generators and let $w$ be a reduced word in $F$. For $x \in F$ let $N_{w}(x)$ be the number of copies of $w$ in $x$ when $x$ is written as a cylically reduced word and let $H_{w}(x)=N_{w}(x)-N_{w^{-1}}(x)$. Note that $H_{w}(-x)=-H_{w}(x)$ and $H_{w}\left(w^{n}\right)=n$. Brooks proved the following:

Theorem 2.1. The function $H_{w}$ is an anti-symmetric quasi-morphism and if $w$ is not a power of $a$ or $b$ then it is not a bounded distance from a homomorphism. If $w$ is cyclically reduced, then $H_{w}\left(w^{n}\right) \geq n$.

Note that if $N$ is a finite group then the Brooks quasi-morphisms can be extended to $F \times N$ by choosing them to be constant on the second factor.

In [5], Dahmani-Guirardel-Osin show that an acylindrically hyperbolic group $G$ contains a copy of a hyperbolically embedded $F \times N$ where $N$ is the maximal finite normal subgroup of $G$. In [6], Hull-Osin show that any antisymmetric quasi-morphism on a hyperbolically embedded subgroup extends to a quasi-morphism of the entire group. Combing these two results we have the following theorem. 
Theorem 2.2. Let $G$ be acylindrically hyperbolic. Then there exists a free group $F=\langle a, b\rangle<G$ such that for every Brooks quasi-morphism $H_{w}$ there is a quasi-morphism $H: G \rightarrow \mathbb{R}$ such that $\left.H\right|_{F}=H_{w}$.

Remark 2.3. There is a weaker version of this theorem (that would be good enough for our applications here) that follows from [2]. The approach in [2] is more direct as it does not go through the theory of hyperbolically embedded subgroups. We also note that both approaches use the projection complex from [1] in an essential way.

As a demonstration of our methods we first give a proof of Theorem 1.3 .

Proof. Given a quasi-morphism $H$ with the defect $\Delta$ and an element $g=$ $g_{1} \ldots g_{n}$ by repeatedly applying the quasi-morphism bound we have

$$
\left|H(g)-\sum_{i=1}^{n} H\left(g_{i}\right)\right| \leq(n-1) \Delta .
$$

If $g=w\left(g_{1}, \ldots, g_{k}\right)$ and $H$ is anti-symmetric this becomes

$$
\left|H(g)-\sum_{i=1}^{n} e_{i} H\left(g_{i}\right)\right| \leq(|w|-1) \Delta
$$

and when $d(w)=0$ (so all the $e_{i}=0$ ) this becomes $|H(g)| \leq(|w|-1) \Delta$ for $g \in w[G]$. More generally for $g \in w(G)$ we have $|H(g)| \leq(v l(g)|w|-1) \Delta$ and therefore if $|H(g)|>0$ we have $\operatorname{svl}(g)>0$ since $H\left(g^{n}\right) \geq n H(g)$ for all $n>0$.

We will use the Brooks construction (and the Hull-Osin extension) to find a $g \in w(G)$ with $H_{g}\left(g^{n}\right) \geq n$. To do this we need to find a cyclically reduced word in $w(F) \subset w(G) \cap F$. Pick a non-trivial element $h \in w(F)$. If it is cyclically reduced let $g=h$ and we are done. If not, then $h=a \cdots a^{-1}$ for a basis element $a$ (or its inverse). Let $h^{\prime}$ be obtained from $h$ by swapping $a$ 's and $b$ 's (with $b$ another basis element). Then $h h^{\prime}$ is cyclically reduced and still in $w(F)$ so $g=h h^{\prime}$ is the desired element.

If $w \in[G, G]$ then for any $g \in w(G), \operatorname{cl}(g) \leq \operatorname{cl}(w) v l(g)$ so $\operatorname{scl}(g) \leq$ $\operatorname{cl}(w) \operatorname{svl}(g)$. In particular if $\operatorname{scl}(g)>0$ then $\operatorname{svl}(g)>0$ and Theorem 1.3 would follow if we knew that every verbal subgroup of an acylindrically hyperbolic group had an element $g$ with $\operatorname{scl}(g)>0$. However, proving this does not seem any easier that the more general proof above.

One can also ask if $\operatorname{scl}(g)=0$ implies that $\operatorname{svl}_{w}(g)=0$ for all $w$. Here the answer is negative. For example, take $w=[[x, y],[z, u]]$ and

$$
G=\left\langle a, b, c, d, t \mid t[[a, b],[c, d]] t^{-1}=[[a, b],[c, d]]^{-1}\right\rangle
$$


Then for $g=[[a, b],[c, d]]$ we have that $g$ is conjugate to $g^{-1}$, which forces $\operatorname{scl}(g)=0$. On the other hand, we claim that $s v l_{w}(g)>0$. Indeed, if $\operatorname{svl}_{w}(g)=0$, then $g^{n}$ can be written as a product of a sublinear number of double commutators, which would imply that $\operatorname{scl}_{H}(g)=0$ where $H=[G, G]$. We now argue that $\operatorname{scl}_{H}(g)>0$. In fact we will show that there is an index 2 subgroup $N<G$ with $H<N$ and so that $N$ surjects to the free group $F_{4}=\langle a, b, c, d\rangle$ with $g$ mapping to $[[a, b],[c, d]]$. Since nontrivial elements in free groups have positive $s c l$ the claim follows. In fact, it $\operatorname{shows~} \operatorname{sl}_{N}(g)>0$. It immediately implies $\operatorname{scl}_{H}(g)>0$.

We let $N$ be the kernel of $G \rightarrow \mathbb{Z} / 2$ that sends $t$ to 1 and $a, b, c, d$ to 0 . The corresponding double cover $Y$ of the presentation 2-complex $X$ of $G$ consists of the disjoint union of two roses $R_{i}$ with petals labeled $a_{i}, b_{i}, c_{i}, d_{i}, i=$ 1,2 , with edges $t_{i}$ connecting the vertex of $R_{i}$ to the vertex of $R_{3-i}$. The map to $X$ is the obvious one, sending $a_{i}$ to $a$ etc. The relation 2-cell in $X$ lifts to two 2-cells in $Y$, with attaching maps $t_{i}\left[\left[a_{i+1}, b_{i+1}\right],\left[c_{i+1}, d_{i+1}\right]\right] t_{i}^{-1}\left[\left[a_{i}, b_{i}\right]\left[c_{i}, d_{i}\right]\right]$ with indices taken mod 2. Now map the 1-skeleton of $Y$ to the rose corresponding to $\langle a, b, c, d\rangle$ via $t_{i} \mapsto *, a_{1}, c_{2} \mapsto a, b_{1}, d_{2} \mapsto b, c_{1}, a_{2} \mapsto$ $c, d_{1}, b_{2} \mapsto d$. We then extend this to the two 2-cells. This is possible since via the attaching maps the boundary of the 2-cells are mapped to $[[c, d],[a, b]][[a, b],[c, d]]$ and $[[a, b],[c, d]][[c, d],[a, b]]$, which are trivial in $\langle a, b, c, d\rangle$ since $[x, y]^{-1}=[y, x]$.

\section{Some facts about the Hull-Osin extension}

Unfortunately, rather than just the statement of Theorem 2.2 we need some elements of the proof in [6]. We review them now. In this section $F$ can be any hyperbolically embedded subgroup in $G$.

It is convenient to replace the quasi-morphism with a function on $G \times G$, called a bicombing in [6]. If $H$ is a quasi-morphism we define $r(x, y)=$ $H\left(x^{-1} y\right)$. Note that $r(z x, z y)=r(x, y)$ and $|r(x, y)+r(y, z)+r(z, x)|$ is bounded by the defect of $H$. On the other hand if we are given a map $r(x, y)$ (satisfying the properties from the previous sentence) then the map $x \mapsto r(1, x)$ is a quasi-morphism so $r$ determines $H$ just as $H$ determines $r$. In particular, to construct $\tilde{H}$, in [6] they first construct $\tilde{r}: G \times G \rightarrow$ $\mathbb{R}$. To construct $\tilde{r}$ for each $x, y \in G$ and each coset $a F$ is associated a finite collection of pairs $E(x, y ; a F)=\left\{\left(x_{i}, y_{i}\right)\right\}$ where $x_{i}, y_{i} \in F$. For the convenience of the reviewer we briefly review the construction of the sets and then state the key properties that we will need.

Let $\Gamma$ be a Cayley graph for $G$ formed from a generating set that contains 
every element of $F$. Given $x, y \in G$ let $\gamma$ be a geodesic in $\Gamma$ from $x$ to $y$. Each $F$-coset has diameter one in $\Gamma$ so $\gamma$ will intersect a given coset $a F$ in at most two points $x^{\prime}$ and $y^{\prime}$. We say that $\gamma$ essentially penetrates $a F$ if any path in $\Gamma$ from $x^{\prime}$ to $y^{\prime}$ that doesn't contain any $F$-edges has length $\geq C$ where $C$ is a constant that only depends on $G$ and $F$. We let $S(x, y)$ be the set of cosets $a F$ where there is some geodesic from $x$ to $y$ that essentially penetrates $a F$. A central fact from [6] is that if there is one geodesic that essentially penetrates then every geodesic from $x$ to $y$ must intersect $a F$. For each coset in $a F \in S(x, y)$ we let $E(x, y ; a F)$ be the set of pairs $\left(x^{\prime}, y^{\prime}\right) \in F$ such that $a x^{\prime}$ and $a y^{\prime}$ are the entry and exit points for a geodesic from $x$ to $y$ in $\Gamma$. For each coset the particular representative $a$ is not important except that the choice needs to be fixed for once and all. If $a F \notin S(x, y)$ then $E(x, y ; a F)$ is empty.

We now define

$$
\tilde{r}(x, y)=\sum_{a F \in S(x, y)}\left(\frac{1}{|E(x, y ; a F)|} \sum_{\left(x^{\prime}, y^{\prime}\right) \in E(x, y ; a F)} r\left(x^{\prime}, y^{\prime}\right)\right) .
$$

For this to be well defined we need the sum to be finite. The inside sum is finite by Lemma 3.8 of [6] and the outside sum is finite since $S(x, y)$ is finite by Corollary 3.4. Note that while in [6] it is only stated that the size of $E(x, y ; a F)$ is finite it is in fact uniformly bounded which will be important in the proof of Lemma 4.1 later.

The following lemma is a combination of Lemma 3.9 and (the proof of) Lemma 4.7 in [6]. We fix the word metric with respect to a finite generating set on $F$ and denote the distance between $x, y$ by $|x-y|$.

Lemma 3.1. Given $x, y, z \in G$ for all but at most two cosets aF exactly one of the following three possibilities holds:

1. $E(x, y ; a F)=\emptyset$;

2. $E(x, y ; a F)=E(x, z ; a F) \neq \emptyset$ and $E(y, z ; a F)=\emptyset$;

3. $E(x, y ; a F)=E(y, z ; a F) \neq \emptyset$ and $E(x, z ; a F)=\emptyset$.

If aF doesn't satisfy the above then either

(A) All of $E(x, y ; a F), E(x, z ; a F)$ and $E(y, z ; a F)$ are non-empty and for any pairs $\left(x^{\prime}, y^{\prime}\right) \in E(x, y ; a F),\left(x^{\prime \prime}, z^{\prime}\right) \in E(x, z ; a F)$ and $\left(y^{\prime \prime}, z^{\prime \prime}\right) \in$ $E(y, z ; a F),\left|x^{\prime}-x^{\prime \prime}\right|,\left|y^{\prime}-y^{\prime \prime}\right|$ and $\left|z^{\prime}-z^{\prime \prime}\right|$ are uniformly bounded. 
(B) Only $E(x, z ; a F)$ is empty and for any pairs $\left(x^{\prime}, y^{\prime}\right) \in E(x, y ; a F)$ and $\left(z^{\prime}, y^{\prime \prime}\right) \in E(z, y ; a F),\left|x^{\prime}-z^{\prime}\right|$ and $\left|y^{\prime}-y^{\prime \prime}\right|$ are uniformly bounded or the same statement holds with $y$ and $z$ swapped.

(C) Only $E(x, y ; a F)$ is non-empty and for all pairs $\left(x^{\prime}, y^{\prime}\right) \in E(x, y ; a F)$, $\left|x^{\prime}-y^{\prime}\right|$ is uniformly bounded.

Given a pair $(x, y) \in G$ let $B(x, y)$ be the collection of cosets that don't satisfy (1)-(3) and let $B(x, y, z)$ be the union of $B(x, y), B(y, z)$ and $B(z, x)$. By Lemma 3.1, $B(u, v)$ contains at most 2 cosets so $B(x, y, z)$ contains at most six. Cosets in $B(x, y, z)$ are of type (A), (B) or (C) depending on which of the conditions in Lemma 3.1 they satisfy.

We are interested in the sum $\tilde{r}(x, y)+\tilde{r}(y, z)+\tilde{r}(z, x)$. It will be convenient to define new sets $E(x, y, z ; a F)$ to be the product of the sets of pairs $E(x, y ; a F), E(y, z ; a F)$ and $E(z, x ; a F)$. Note that one or more of the sets may be empty in which case the product would be empty. (In fact for at most one coset at least one of the sets will be empty.) To get around this if $E(u, v ; a F)$ is empty we make it non-empty by adding the "empty pair" $(\emptyset, \emptyset)$ and we define $r(\emptyset, \emptyset)=0$. With this modification $E(x, y, z ; a F)$ will always be a triple of pairs in $F \cup\{\emptyset\}$. Next we define

$$
\rho(x, y, z ; a F)=\frac{1}{|E(x, y, z ; a F)|} \sum_{E(x, y, z ; a F)} r\left(x_{-}, y_{+}\right)+r\left(y_{-}, z_{+}\right)+r\left(z_{-}, x_{+}\right)
$$

and observe that

$$
\tilde{r}(x, y)+\tilde{r}(y, z)+\tilde{r}(z, x)=\sum_{a F} \rho(x, y, z ; a F) .
$$

To show that $\tilde{r}$ determines a quasi-morphism Hull-Osin show that for nearly all cosets the expression $\rho(x, y, z ; a F)$ is zero and for the finitely many when it is not it is uniformly bounded.

Corollary 3.2. If $a F \notin B(x, y, z)$ then $\rho(x, y, z ; a F)=0$.

Proof. If $a F \notin B(x, y, z)$ then either $E(x, y, z ; a F)$ is the triple of empty pairs and $\rho(x, y, z ; a F)=0$ or all the terms in the sum cancel and again $\rho(x, y, z ; a F)=0$.

\section{Many independent quasi-morphisms}

For the remainder of the paper we can assume that $d(w)>1$. If $H: G \rightarrow \mathbb{Z}$ is a homomorphism, an easy calculation gives that for any $g \in w(G)$ we 
have that $H(g)$ is divisible by $d(w)$. We will construct a family of quasimorphisms where this is true for nearly all the quasi-morphisms in the family where the number of exceptions is bounded above by the $v l(g)$.

Let $F \times N$ be hyperbolically embedded in $G$ where $F$ is the free group of rank at least two and $N$ is a finite group. For simplicity we suppose the rank of $F$ is two in the following. We now fix a sequence of words that we will use to build Brooks' quasi-morphisms on $F$, then extend it to $F \times N$, trivially on $N$. Let $g_{i}^{\prime}=a b^{2 i}, g_{i}^{\prime \prime}=a b^{2 i+1}$ and $g_{i}=g_{i}^{\prime} g_{i}^{\prime \prime}$ and let $H_{i}=H_{g_{i}}$ be the Brooks quasi-morphism and $r_{i}$ the corresponding bicombings.

We fix the word metric with respect a finite generating set on $F \times N$ and denote the distance between $x, y$ by $|x-y|$.

Lemma 4.1. Given a triple of pairs $\left(x_{-}, x_{+}\right),\left(y_{-}, y_{+}\right),\left(z_{-}, z_{+}\right)$in $F \times N$ with $\left|x_{-}-x_{+}\right|,\left|y_{-}-y_{+}\right|$and $\left|z_{-}-z_{+}\right|$bounded by $L$ there are at most

- L of the $r_{i}$ such that $r_{i}\left(x_{-}, x_{+}\right) \neq 0$;

- $2 L$ of the $r_{i}$ such that $r_{i}\left(x_{-}, y_{+}\right)+r_{i}\left(y_{-}, x_{+}\right) \neq 0$;

- $3 L+3$ of the $r_{i}$ such $r_{i}\left(x_{-}, y_{+}\right)+r_{i}\left(y_{-}, z_{+}\right)+r_{i}\left(z_{-}, x_{+}\right) \neq 0$.

Therefore there is a uniform bound on the number of $\rho_{i}$ where $\rho_{i}(x, y, z ; a F) \neq$ 0 .

Proof. We only discuss the case that $N$ is trivial. The general case is similar.

If $r_{i}\left(x_{-}, x_{+}\right) \neq 0$ there is a translate of the word $g_{i}$ in the segment between $x_{-}$and $x_{+}$in the Cayley graph (with the standard generators). Since two $g_{i}$ can't intersect in a segment (a very bad) upper bound for the number of $r_{i}$ with $r_{i}\left(x_{-}, x_{+}\right) \neq 0$ is $\left|x_{-}-x_{+}\right| \leq L$.

The triple $x_{-}, x_{+}$and $y_{+}$form a tripod in the Cayley graph and let $m$ be the central vertex. Then $r_{i}\left(x_{-}, y_{+}\right)+r_{i}\left(y_{+}, x_{+}\right)=0$ unless there is a a translate of $g_{i}$ in the segment from $y_{+}$to $x_{-}$that intersects the segment from $m$ to $x_{-}$or a translate in the segment from $y_{+}$to $x_{+}$that intersects the segment from $m$ to $x_{+}$. Again using the fact that two $g_{i}$ 's can't overlap in a segment an upper bound for the number of $r_{i}$ 's with $r_{i}\left(x_{-}, y_{+}\right)+r_{i}\left(y_{+}, x_{+}\right) \neq 0$ is $\left|x_{-}-m\right|+\left|x_{+}-m\right|=\left|x_{-}-x_{+}\right| \leq L$. Similarly there are at most $L$ of the $r_{i}$ such that $r_{i}\left(x_{+}, y_{+}\right)+r_{i}\left(y_{+}, x_{+}\right) \neq 0$ or equivalently $r_{i}\left(y_{+}, x_{+}\right)=r_{i}\left(y_{-}, x_{+}\right)$for all but $L$ of the $r_{i}$ and therefore $r_{i}\left(x_{-}, y_{+}\right)+r_{i}\left(y_{-}, x_{+}\right)=0$ for all but $2 L$ of the $r_{i}$.

Now we examine the tripod formed by $x_{-}, y_{-}$and $z_{-}$. As with the original Brooks' argument the sum

$$
r_{i}\left(x_{-}, y_{-}\right)+r_{i}\left(y_{-}, z_{-}\right)+r_{i}\left(z_{-}, x_{-}\right)
$$


is zero unless the a translate of the word $g_{i}$ intersects the central vertex of the tripod. At most three such words can intersect the central vertex so the sum is non-zero for at most 3 of the $r_{i}$. As above for at most $L$ of the $r_{i}$ we have $r_{i}\left(x_{-}, y_{-}\right)=r_{i}\left(x_{i}, y_{+}\right)$, etc. Therefore

$$
r_{i}\left(x_{-}, y_{+}\right)+r_{i}\left(y_{-}, z_{+}\right)+r_{i}\left(z_{-}, x_{+}\right)=0
$$

for all but at most $3 L+3$ of the $r_{i}$.

Since $F$ is hyperbolically embedded in $G$, if $a F \notin B(x, y, z)$ then $\rho_{i}(x, y, z ; a F)=$ 0 for all $i$ by Corollary 3.2. For cosets $a F \in B(x, y, z)$ of type (A) there will be at most $(3 L+3)|E(x, y, z ; a F)|$ of the $\rho_{i}$ with $\rho_{i}(x, y, z ; a F) \neq 0$, for cosets of type (B) at most $2 L|E(x, y, z ; a F)|$ and for cosets of type (C) at most $L|E(x, y, z ; a F)|$. By Lemma 3.8 of [6] $|E(u, v ; a F)|$ is uniformly bounded 1 and therefore so is $|E(x, y, z ; a F)|$. It follows that there is uniform bound on the number of $\rho_{i}$ with $\rho_{i}(x, y, z ; a F) \neq 0$.

Since $F \times N$ is hyperbolically embedded in $G$, let $\tilde{H}_{i}: G \rightarrow \mathbb{R}$ be the Hull-Osin extension of the $H_{i}$ and $\tilde{r}_{i}$ the corresponding bicombings.

Proposition 4.2. There exists an $M>0$ such that for any $x, y \in G$,

$$
\tilde{H}_{i}(x y)-\tilde{H}_{i}(x)-\tilde{H}_{i}(y)=0
$$

holds except for at most $M$ of the $\tilde{H}_{i}$. It follows that for any $a_{1}, \cdots a_{k} \in G$,

$$
\tilde{H}_{i}\left(a_{1} \cdots a_{k}\right)-\tilde{H}_{i}\left(a_{1}\right)-\cdots-\tilde{H}_{i}\left(a_{k}\right)=0
$$

holds except for at most $M(k-1)$ of the $\tilde{H}_{i}$.

Proof. First observe

$$
\tilde{H}_{i}(x y)-\tilde{H}_{i}(x)-\tilde{H}_{i}(y)=\tilde{r}_{i}(i d, x y)+\tilde{r}_{i}(x, i d)+\tilde{r}_{i}(x y, x)
$$

so we can instead show that

$$
\tilde{r}_{i}(x, y)+\tilde{r}_{i}(y, z)+\tilde{r}_{i}(z, x)=0
$$

for all but $M$ of the $\tilde{r}_{i}$. But this follows from Lemma 4.1, as for all but at most 6 cosets $\rho_{i}(x, y, z ; a F)=0$ for all $i$ and for each of these bad cosets there is a uniform bound on the number of $\rho_{i}$ with $\rho_{i}(x, y, z ; a F) \neq 0$.

\footnotetext{
${ }^{1}$ In [6. Lemma 3.8] it is only claimed that $|E(x, y ; a F)|$ is finite however it is easy to see that their proof shows that the bound is uniform since the constant $C$ in Lemma 3.3/2.4 is uniform.
} 
Lemma 4.3. For each $K$ there exists $g \in w(F \times N)$ such that $H_{i}(g)=1$ for all $1 \leq i \leq K$.

Proof. We will find an element $g \in w(F)$. Recall that we are assuming that $d=d(w)>1$ and therefore for any $f \in F$, we have $f^{d} \in w[F]$. Let $h_{i}^{\prime}=\left(g_{i}^{\prime}\right)^{d}$ and $h_{i}^{\prime \prime}=\left(g_{i}^{\prime \prime}\right)^{d}$. Then the product $h_{i}=h_{i}^{\prime} h_{i}^{\prime \prime}$ contains a single copy of $g_{i}$. Let $g=h_{1} h_{2} \cdots h_{K}$. Note that $g$ is already reduced since there are only positive powers of $a$ and $b$ in the $h_{i}^{\prime}$ and $h_{i}^{\prime \prime}$. Furthermore by our construction of the $g_{i}$ there will be exactly one copy of $g_{i}$ in $g$ and no copies of $g_{i}^{-1}$. Therefore $H_{i}(g)=1$ for $1 \leq i \leq K$.

We now give a proof of Theorem 1.1 when $d(w)>1$.

Proof. We first show that for any $g \in w(G)$ we have that $\tilde{H}_{i}(g)$ is divisible by $d(w)>1$ for all but a bounded number of the $\tilde{H}_{i}$ where the bound only depends on $v l(g)$. To see this we first observe that if $g=w\left(g_{1}, \ldots, g_{k}\right) \in$ $w[G]$ then by Proposition 4.2

$$
\tilde{H}_{i}\left(w\left(g_{1}, \cdots, g_{k}\right)\right)=\sum_{j=1}^{k} e_{j} \tilde{H}_{i}\left(g_{j}\right),
$$

for all but $M(|w|-1)$ of the $\tilde{H}_{i}$. In particular, for any $g \in w[G]$ there are at most $M(|w|-1)$ of the $i$ such that $\tilde{H}_{i}(g)$ isn't divisible by $d(w)$. Similarly if $g \in w(G)$ is product of $v l(g)$ elements $g_{j} \in w[G]$ then

$$
\tilde{H}_{i}(g)=\sum_{j=1}^{v l(g)} \tilde{H}_{i}\left(g_{j}\right)
$$

for all but $M(v l(g)-1)$ of the $\tilde{H}_{i}$. If all of the $\tilde{H}_{i}\left(g_{j}\right)$ are divisible by $d(w)$ then so is $\tilde{H}_{i}(g)$ so we have that $\tilde{H}_{i}(g)$ is divisible by $d(w)$ for all but at most $M(v l(g)-1)+v l(g)(M(|w|-1))$ of the $\tilde{H}_{i}$. In particular a bound on $v l(g)$ gives a bound on the number of $\tilde{H}_{i}$ where $\tilde{H}_{i}(g)$ is not divisible by $d(w)>1$.

On the other hand, by Lemma 4.3, for any $K>0$ we can find a word $h_{K}$ such that $\tilde{H}_{i}\left(h_{K}\right)=H_{i}\left(h_{K}\right)=1$ for $1 \leq i \leq K$. Therefore $v l\left(h_{K}\right) \rightarrow \infty$ as $K \rightarrow \infty$.

From the above proof we see that $v l\left(h_{K}\right) \geq K /(M|w|)$. We know of no examples where this bound is sharp. 


\section{References}

[1] Mladen Bestvina, Ken Bromberg, and Koji Fujiwara. Constructing group actions on quasi-trees and applications to mapping class groups. Publications mathématiques de l'IHÉS, pages 1-64, 2014.

[2] Mladen Bestvina, Kenneth Bromberg, and Koji Fujiwara. Projection complexes, acylindrically hyperbolic groups and bounded cohomology. http://arxiv.org/abs/1306.1542.

[3] Brian H. Bowditch. Tight geodesics in the curve complex. Invent. Math., 171(2):281-300, 2008.

[4] Danny Calegari and Dongping Zhuang. Stable $W$-length. In Topology and geometry in dimension three, volume 560 of Contemp. Math., pages 145-169. Amer. Math. Soc., Providence, RI, 2011.

[5] F. Dahmani, V. Guirardel, and D. Osin. Hyperbolically embedded subgroups and rotating families in groups acting on hyperbolic spaces. Memoirs of the AMS, to appear. arXiv:1111.7048.

[6] Michael Hull and Denis Osin. Induced quasicocycles on groups with hyperbolically embedded subgroups. Algebr. Geom. Topol., 13(5):2635$2665,2013$.

[7] Alexei Myasnikov and Andrey Nikolaev. Verbal subgroups of hyperbolic groups have infinite width. J. Lond. Math. Soc. (2), 90(2):573-591, 2014.

[8] D. Osin. Acylindrically hyperbolic groups. Trans. Amer. Math. Soc., 368(2):851-888, 2016.

[9] A. H. Rhemtulla. A problem of bounded expressibility in free products. Proc. Cambridge Philos. Soc., 64:573-584, 1968.

[10] Dan Segal. Words: notes on verbal width in groups, volume 361 of London Mathematical Society Lecture Note Series. Cambridge University Press, Cambridge, 2009. 